\title{
$\mathbf{J}|\mathbf{A}| \mathbf{C} \mid \mathbf{S}$ \\ COMMUNICATIONS
}

Published on Web 10/17/2002

\section{Efficient Mixing and Reactions within Microfluidic Channels Using Microbead-Supported Catalysts}

\author{
Gi Hun Seong and Richard M. Crooks* \\ Department of Chemistry, Texas A\&M University, P.O. Box 30012, College Station, Texas 77842-3012
}

Received July 8, 2002

We report a novel strategy for efficiently mixing solutions and carrying out multistep catalytic reactions in microfluidic systems. The approach involves immobilizing catalysts on microbeads, placing the beads into well-defined microreactor zones, and then passing reactants through one or more of the reactor zones to yield products. The catalyst-modified beads mix reactants and increase the effective surface area of the channel interior, both of which improve reaction velocities compared to open channels. In addition to providing a general route to chemical synthesis within microfluidic systems, this design strategy may also be applicable to modeling reaction pathways within cells and for bio/chemical sensing applications. ${ }^{1-4}$

In open microchannels having dimensions on the order of 100 $\mu \mathrm{m}$, mass transfer is dominated by laminar flow, and thus mixing is driven principally by diffusion. ${ }^{5,6}$ However, the diffusional time scale is often too long for technological applications such as synthesis and bio/chemical sensing. This problem has most often been addressed by using either slow flow rates or long channels, neither of which is optimal. We have discovered that interstices between microbeads packed within channels provide a simple means for reducing the effective thickness of fluid laminae, thereby greatly increasing the mixing rate for fluids. Moreover, the microbeads themselves have a high surface area relative to that of the channel walls; thus, they also act as a convenient support for heterogeneous catalysts. ${ }^{7}$ Herein we demonstrate both of these functions.

The principles of laminar shear flow, extensional flow, and distributive mixing are relevant for efficient mixing under conditions of laminar flow. ${ }^{8}$ Laminar shear flow and extensional flow lead to an increase in interfacial area of the fluid elements and a sufficient reduction in the thickness of the fluid laminae that molecular diffusion alone results in rapid mixing. Distributive mixing physically splits fluid streams into smaller segments and redistributes them in such a way that the striation thickness is significantly reduced. Several approaches for mixing confluent liquid streams have recently been reported. For example, Stroock et al. ${ }^{9}$ presented a general strategy for creating transverse flow in microchannels that can be used to induce chaotic stirring at low Reynolds number ( $R e$, which is proportional to the ratio of the inertial and viscous forces). Johnson et al. ${ }^{10}$ fabricated a series of slanted wells within a polycarbonate sheet to induce rapid lateral transport of two confluent streams. He et al. ${ }^{11}$ described mixing of liquids transported by electroosmotic flow in a microfabricated device having multiple intersecting channels of varying length and a bimodal width distribution. This problem has also been studied using numerical simulations. ${ }^{12}$

A schematic diagram of the first microfluidic system used in this study is shown in Figure 1A. The device was fabricated from poly(dimethylsiloxane) (PDMS) using standard photolithographic and replica molding methods. ${ }^{13}$ It consists of two $100 \mu \mathrm{m}$-wide

* To whom correspondence should be addressed. E-mail: crooks@tamu.edu.

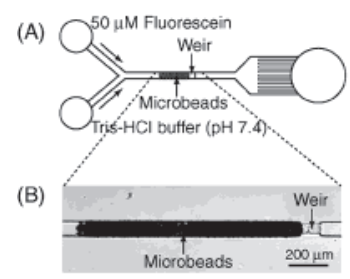

(C)

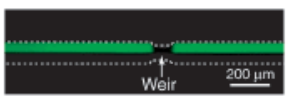

(D)

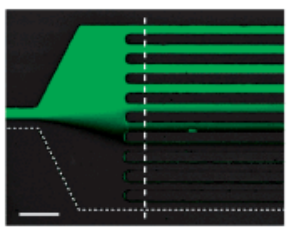

(E)

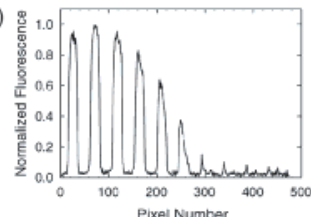

(F)

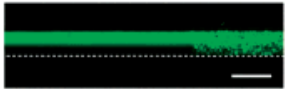

(G)

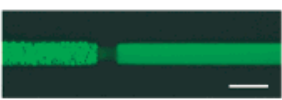

$(\mathrm{H})$

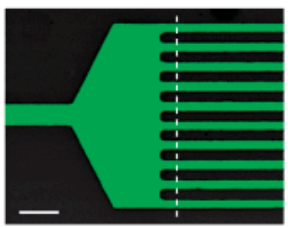

(1)

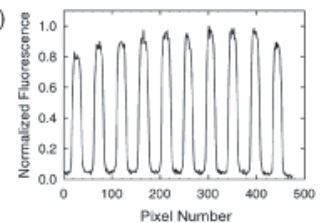

Figure 1. (A) Schematic illustration of the PDMS microdevice used for studying two-stream mixing. (B) Optical image of microbeads packed in the microreactor. (C) Fluorescence micrograph (FM) of the weir in the open channel. (D) FM showing the 10-lane detection zone after flow through the open channel. (E) Fluorescence intensity line scans at the location indicated by the dashed line in (D). (F) FM of the microbead-bed inlet stream. (G) FM showing the mixed fluid stream exiting the microbead bed. (H) FM showing the 10-lane detection zone after flow through the microbead-containing channel. (I) Fluorescence intensity line scans at the location indicated by the dashed line in $(\mathrm{H})$. Excitation wavelength: 494 $\mathrm{nm}$; maximum emission wavelength: $520 \mathrm{~nm}$. The flow rate was $0.5 \mu \mathrm{L} /$ min in all cases.

inlet channels, a main channel containing a weir, and ten $50 \mu \mathrm{m}$ wide spatially addressable detection lanes at the end of the main channel. All channels were $23 \mu \mathrm{m}$ deep. Polystyrene microbeads (Bangs Laboratories, Fishers, IN) were introduced into the main channel by hand pumping in less than $30 \mathrm{~s}$. As shown in Figure $1 \mathrm{~B}$, the $15.5 \mu \mathrm{m}$-diameter beads were retained by the $7-12 \mu \mathrm{m}$ deep weir. ${ }^{14}$ To demonstrate the mixing efficiency of the beads, a $50 \mu \mathrm{M}$ fluorescein solution in Tris- $\mathrm{HCl}(\mathrm{pH}$ 7.4) buffer and buffer only were pumped through the two inlet channels at a flow rate of $0.5 \mu \mathrm{L} / \mathrm{min}$. Prior to bead packing, laminar flow of the two solutions through the channel is not significantly disrupted by the weir structure. Evidence for this comes from fluorescence micrographs of the postweir portion of the channel (Figure 1C) and the 10-lane detection zone of the device (Figure 1, D and E). ${ }^{15,16}$

Microbeads introduced into the channel of the device illustrated in Figure 1A promote both laminar and turbulent mixing. Figure 
(A)

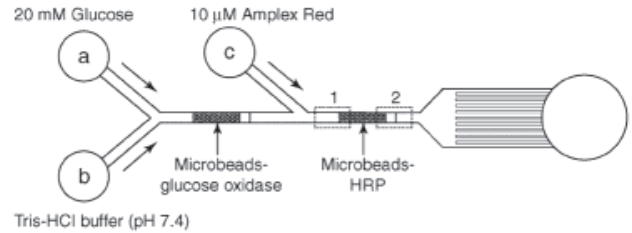

(B)
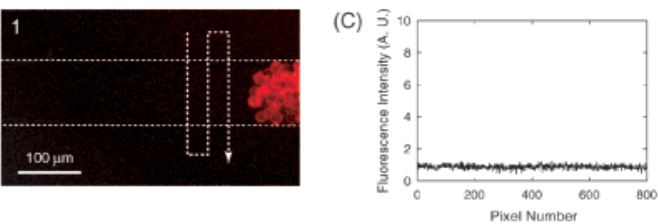

(D)
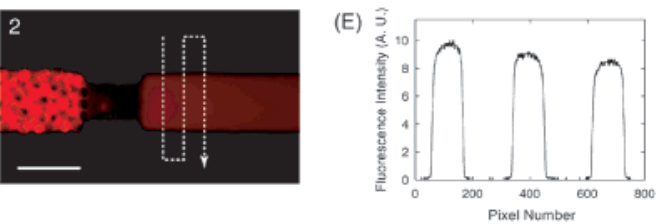

Figure 2. (A) Schematic illustration of the microdevice used to demonstrate multiple, sequential reactions. (B) FM of the inlet stream just before it enters the HRP microreactor (region 1 in (A)). (C) Fluorescence intensity line scans at the locations indicated by the dashed line in (B). (D) FM of the outlet stream just after exiting the HRP microreactor (region 2 in (A)). (E) Fluorescence intensity line scans at the locations indicated by the dashed line in (D). Excitation wavelength: $563 \mathrm{~nm}$; maximum emission wavelength: $587 \mathrm{~nm}$. The flow rate was $0.5 \mu \mathrm{L} / \mathrm{min}$ in all cases.

$1 \mathrm{~F}$ shows the buffer-only and buffer-plus-dye laminae in the main channel prior to the microbead bed, while Figure $1 \mathrm{G}$ shows the mixed solution just after the flow stream encounters the $1.1 \mathrm{~mm}-$ long bed of microbeads. Figure $1 \mathrm{H}$ is a fluorescence micrograph of the 10-lane detection zone after mixing, and Figure 1I is a line scan across those lanes. The average normalized fluorescence and standard deviation across the 10-lane detection zone is $0.91 \pm 0.06$. In contrast, the open-channel control experiment (Figure 1E) leads to the type of profile expected for slight mixing at the interface of the two flow streams. While we have not examined all parameters controlling mixing efficiency (flow rate, bead size, channel dimensions, bead bed length), it is true that longer packed beds of beads result in better mixing.

Effective mixing increases the velocity of diffusion-controlled reactions. To demonstrate this point, we attached enzymes to the microbeads prior to packing them into the microfluidic channel and followed the resulting substrate reactions using a fluorescence microscope equipped with a standard CCD digital camera. The approach to this experiment is illustrated schematically in Figure 2A. Biotin-labeled glucose oxidase (GOX) and horseradish peroxidase (HRP) were conjugated onto microbeads coated with streptavidin. These microbeads were subsequently packed into each of the two discreet reaction zones shown in Figure 2A. Next, aqueous solutions containing $20 \mathrm{mM}$ glucose, $50 \mathrm{mM}$ Tris- $\mathrm{HCl}$ buffer (pH 7.4), and $10 \mu \mathrm{M}$ amplex red (Molecular Probes Inc., Eugene, OR) in $50 \mathrm{mM}$ Tris-HCl buffer (pH 7.4) were introduced into the main channel at a flow rate of $0.5 \mu \mathrm{L} / \mathrm{min}$ from inlet reservoirs $a, b$, and $c$, respectively.

In the first reaction zone, the glucose and buffer streams are mixed by the microbeads, and simultaneously glucose is converted to gluconic acid and $\mathrm{H}_{2} \mathrm{O}_{2}$. Nonfluorescent amplex red is then added to the $\mathrm{H}_{2} \mathrm{O}_{2}$-containing product of the first reaction as a confluent stream. In the second microreactor, fluorescent resorufin is formed via the HRP-catalyzed reaction between $\mathrm{H}_{2} \mathrm{O}_{2}$ and amplex red. ${ }^{16,17}$

A fluorescence image of the main channel at the inlet to the
HRP-functionalized microbeads (region 1 of Figure 2A) is shown in Figure $2 \mathrm{~B}$ and the corresponding line scan is shown in Figure $2 \mathrm{C}$. These data reveal only a very low level of background fluorescence. When the confluent streams containing $\mathrm{H}_{2} \mathrm{O}_{2}$ and amplex red encounter the HRP-labeled microbeads, the two streams efficiently mix and the $\mathrm{H}_{2} \mathrm{O}_{2}$ and amplex red are able to react with the HRP catalyst. A fluorescence micrograph of the product stream exiting the HRP reaction zone (region 2 of Figure 2A) is shown in Figure 2D; the corresponding line scans are shown in Figure 2E. The line scan indicates that the solutions are effectively mixed during their encounter with the catalyst-immobilized beads, and that amplex red is converted into resorufin.

In summary, we have demonstrated a remarkably simple but highly effective approach for mixing confluent streams in microfluidic systems. Specifically, the mixing efficiency is $91 \pm 6 \%$ under the conditions used in this work; longer bead-filled beds and slower flow rates are predicted by theory to improve this percentage further. A major advantage of this strategy is that heterogeneous catalysts can be linked to the microbeads, so that the beads carry out the dual function of mixing and acting as catalyst supports. Additionally, microbead immobilization chemistry is well understood, microbeads have a high surface-area-to-volume ratio, and they are very easy to handle in microfluidic systems.

Acknowledgment. We gratefully acknowledge financial support of this work from the U.S. Army Medical Research \& Materiel Command and the Texas Higher Education Coordinating Board through the Texas Advanced Technology Program (0082-1999). We also acknowledge stimulating discussions with Professor Allen J. Bard (University of Texas, Austin) that led to conceptualization of the general idea of performing sequential reactions in microfluidic systems. Some of the instrumentation used to carry out these experiments was provided by the Center for Integrated Microchemical Systems at Texas A\&M University.

Supporting Information Available: Fluorescence micrographs of an experiment analogous to that shown in Figure 2, but in the absence of microbeads. Fluorescence micrographs of the experiment in Figure 2 showing additional locations along the channel (PDF). This material is available free of charge via the Internet at http://pubs.acs.org.

\section{References}

(1) Bard, A. J. Integrated Chemical Systems: A Chemical Approach to Nanotechnology; Wiley: New York, 1994

(2) Schilling, E. A.; Kamholz, A. E.; Yager, P. Anal. Chem. 2002, 74, $1798-$ 1804.

(3) Losey, M. W.; Schmidt, M. A.; Jensen, K. F. Ind. Eng. Chem. Res. 2001 40, 2555-2562.

(4) Fu, A. Y.; Spence, C.; Scherer, A.; Arnold, F. H.; Quake, S. R. Nat Biotechnol. 1999, 17, 1109-1111.

(5) Ismagilov, R. F.; Rosmarin, D.; Kenis, P. J. A.; Chiu, D. T.; Zhang, W.; Stone, H. A.; Whitesides, G. M. Anal. Chem. 2001, 73, 4682-4687.

(6) Kenis, P. J. A.; Ismagilov, R. F.; Takayama, S.; Whitesides, G. M.; Li, S.; White, H. S. Acc. Chem. Res. 2000, 33, 841-847.

(7) Richter, T.; Shultz-Lockyear, L. L.; Oleschuk, R. D.; Bilitewski, U.; Harrison, D. J. Sens. Actuators, B 2002, 81, 369-376.

(8) Harnby, N.; Edwards, M. F.; Nienow, A. W. Mixing in the Process Industries; Butterworth: Oxford, 1985

(9) Stroock, A. D.; Dertinger, S. K. W.; Ajdari, A.; Mezic, I.; Stone, H. A. Whitesides, G. M. Science 2002, 295, 647-651.

(10) Johnson, T. J.; Ross, D.; Locascio, L. E. Anal. Chem. 2002, 74, 45-51.

(11) He, B.; Burke, B. J.; Zhang, X.; Zhang, R.; Regnier, F. E. Anal. Chem. 2001, 73, 1942-1947.

(12) Erickson, D.; Li, D. Langmuir 2002, 18, 1883-1892.

(13) Duffy, D. C.; McDonald, J. C.; Schueller, O. J. A.; Whitesides, G. M. Anal. Chem. 1998, 70, 4974-4984.

(14) Seong, G. H.; Zhan, W.; Crooks, R. M. Anal. Chem. 2002, 74, 33723377 .

(15) Jeon, N. L.; Dertinger, S. K. W.; Chiu, D. T.; Choi, I. S.; Stroock, A. D.; Whitesides, G. M. Langmuir 2000, 16, 8311-8316.

(16) Mao, H.; Yang, T.; Cremer, P. S. Anal. Chem. 2002, 74, 379-385

(17) Zhou, M.; Diwu, Z.; Panchuk-Voloshina, N.; Haugland, R. P. Anal. Biochem. 1997, 253, 162-168.

JA020932Y 